International Journal of Pure and Applied Mathematics

Volume 85 No. 3 2013, 457-463

ISSN: 1311-8080 (printed version); ISSN: 1314-3395 (on-line version)

url: http://www.ijpam.eu

doi: http://dx.doi.org/10.12732/ijpam.v85i3.2

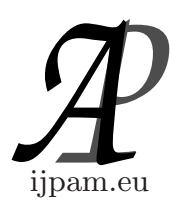

\title{
JULIA SETS OF \\ FINITELY GENERATED RATIONAL SEMIGROUPS
}

\author{
Weifeng Yang \\ Department of Mathematics and Physics \\ Hunan Institute of Engineering \\ Xiangtan, 411104, P.R. CHINA
}

\begin{abstract}
Let $G$ be a semigroup of rational functions with degree at least 2 with the semigroup operation being functional composition. We prove that if $G$ is finitely generated and satisfies $O S C(U)$, then $J(G)$ has no interior points.
\end{abstract}

AMS Subject Classification: 30D05

Key Words: Fatou set, Julia set, rational semigroup

\section{Introduction}

In the series of their papers, Hinkkanen and Martin tried to extend the classical theory of the dynamics associated to the iteration of a rational function of a complex variable to the more general setting of semigroups of rational functions, see $[6,7,8]$ etc.

Let

$$
f_{j}: \hat{\mathbb{C}} \rightarrow \hat{\mathbb{C}}(j=1,2, \cdots)
$$

be non-constant rational functions. We denote by

$$
G=\left\langle f_{1}, f_{2}, \cdots, f_{n}, \cdots\right\rangle
$$

the semigroup generated by the family $\left\{f_{j}: j=1,2, \cdots\right\}$ with the semigroup

Received: October 24, 2012

(c) 2013 Academic Publications, Ltd. url: www.acadpubl.eu 
operation being functional composition, which is called a rational semigroup. If there exists a finite family $\left\{f_{j}: j=1,2, \cdots, m\right\}$ such that $G=\left\langle f_{1}, \cdots, f_{m}\right\rangle$, then we call that $G$ is a finitely generated rational semigroup.

The Fatou set of a rational semigroup $G$ is defined by

$$
F(G)=\{z \in \hat{\mathbb{C}}: \mathrm{G} \text { is normal in some neighbourhood of } \mathrm{z}\}
$$

and the Julia set of $G$ by $J(G)=\hat{\mathbb{C}} \backslash F(G)$. We write $F(f)$ and $J(f)$ for $F(\langle f\rangle)$ and $J(\langle f\rangle)$. Then $F(f)$ and $J(f)$ are the Fatou set and Julia set, respectively, in the classical iteration theory of Fatou-Julia's. It is obvious that the dynamics of a semigroup is more complicated than that of a single function. Many properties in the classical case cannot be preserved for the case of semigroups. For example, $F(G)$ and $J(G)$ may not be complete invariant. In general, we can only know that $F(G)$ is forward invariant and $J(G)$ is backward invariant under $G$. And $J(G)$ may not be $\hat{\mathbb{C}}$ when $J(G)$ has an interior point. See the examples in $[6,22]$.

We refer to $[1,9,15]$ etc for more properties of the iteration of a single rational function and $[2 \sim 8,10 \sim 14,16 \sim 23]$ etc for more properties of the dynamics of rational semigroups.

In the sequel of this note, we always assume that $G$ consists of rational functions with degree at least 2 . We are mainly interested in finitely generated rational semigroups in this paper.

As in the iteration of a single function, the following problem is fundamental and interesting in the study of the dynamics of rational semigroups.

Problem 1.1. When does $J(G)$ contain no interior points if $G$ is finitely generated?

Example 3 in [6] shows that without extra condition(s), $J(G)$ may contain interior points. In order to discuss Problem 1.1, we introduce the following condition.

Definition 1.1. Let $G=\left\langle f_{1}, f_{2}, \cdots, f_{m}\right\rangle$ be a finitely generated rational semigroup. We say that $G$ satisfies the open set condition if there exists an open set $U$ satisfying that $f_{i}^{-1}(U) \subset U$ for each $i(1 \leq i \leq m)$ and $f_{i}^{-1}(U) \cap f_{j}^{-1}(U)=$ $\emptyset$ for each pair $(i, j)$, where $1 \leq i, j \leq m$ and $i \neq j$.

For the sake of simplicity, we always denote the open set condition by $O S C(U)$ in the following.

In Section 2, we will prove the following result.

Theorem 1.1. Let $G=\left\langle f_{1}, f_{2}, \cdots, f_{m}\right\rangle$ be a finitely generated rational semigroup. If $G$ satisfies $O S C(U)$, then the Julia set $J(G)$ of $G$ has no interior 
points.

Remark 1.1. In 2001, Sumi discussed Problem 1.1. As one of the main results in [16], Sumi proved that if $G$ is finitely generated and satisfies $O S C(U)$, and if $U \backslash J(G) \neq \emptyset$, then $J(G)$ has no interior points, see Proposition 4.3 in [16]. Theorem 1.1 shows that the condition " $U \backslash J(G) \neq \emptyset$ " in Sumi's result is unnecessary.

\section{The Proof of Theorem 1.1}

Let $G$ be a rational semigroup. A point $z$ is called exceptional if its backward orbit $O^{-}(z)=\{w$ : there exists an element $g \in G$ such that $g(w)=z\}$ is finite. The set of exceptional points is denoted by $E(G)$. That is,

$$
E(G)=\left\{z \in \hat{\mathbb{C}}: O^{-}(z) \text { contains at most two points }\right\}
$$

Lemma 2.1. (Lemma 3.3 in $[6]) \operatorname{Card}(E(G)) \leq 2$, where "card" means "cardinality".

Let's introduce two more lemmas which are useful for the following proofs.

Lemma 2.2. (Lemma 3.2 in [6]) Let $G$ be a rational semigroup and $z \in \hat{\mathbb{C}} \backslash E(G)$. Then $J(G)$ is contained in the set of accumulation points of $O^{-}(z)$. That is

$$
J(G) \subset\left\{\text { accumulation points of } O^{-}(z)\right\}
$$

Lemma 2.3. (Lemma 1.1.4 (2) in [17]) Let $G=\left\langle f_{1}, f_{2}, \cdots, f_{m}\right\rangle$ be a finitely generated rational semigroup. Then

$$
J(G)=\bigcup_{i=1}^{m} f_{i}^{-1}(J(G)) .
$$

By using Lemmas 2.1 and 2.2, we prove a result which is crucial for the proof of Theorem 1.1.

Lemma 2.4. Let $G=\left\langle f_{1}, f_{2}, \cdots, f_{m}\right\rangle$ be a finitely generated rational semigroup. If there exists an open set $\Omega$ such that $\Omega \cap F(G) \neq \emptyset$ and $f_{i}^{-1}(\Omega \cap$ $F(G)) \subset \Omega \cap F(G)$ for each $i(1 \leq i \leq m)$, then $\operatorname{Int}(J(G))=\emptyset$, where $\operatorname{Int}(J(G))$ denotes the interior of $J(G)$. 
Proof. Without loss of generality, we may assume that $J(G) \neq \emptyset$ and $z \in J(G)$. It follows from $\Omega \cap F(G) \neq \emptyset$ and Lemma 2.1 that there exists $z_{0} \in \Omega \cap F(G)$ such that $z_{0} \notin E(G)$.

Let $W$ be any neighborhood of $z$. Then Lemma 2.2 implies that there is an element $g \in G$ such that $g^{-1}\left(z_{0}\right) \in W$. Since

$$
f_{i}^{-1}(\Omega \cap F(G)) \subset \Omega \cap F(G) \quad(i=1,2, \cdots m),
$$

we see that $g^{-1}\left(z_{0}\right) \in \Omega \cap F(G) \subset F(G)$. Hence $W \cap F(G) \neq \emptyset$. This shows that $z$ is not a interior point of $J(G)$. The proof is completed.

The following result is obvious.

Lemma 2.5. Let $G=\left\langle f_{1}, f_{2}, \cdots, f_{m}\right\rangle$ be a finitely generated rational semigroup. Suppose that $G$ satisfies $O S C(U)$. If we let $V_{i}=f_{i}^{-1}(U)$ and $V=\bigcup_{i=1}^{m} V_{i}$, then $V$ is a proper subset of $U$ and $\bigcup_{i=1}^{m} f_{i}^{-1}(V)$ is a proper subset of $V$.

Lemma 2.6. Let $G=\left\langle f_{1}, f_{2}, \cdots, f_{m}\right\rangle$ be a finitely generated rational semigroup. If $G$ satisfies $O S C(U)$, and if $V_{i}$ and $V$ are as in Lemma 2.5, then $V \cap F(G) \neq \emptyset$ and for each $j(1 \leq j \leq m)$,

$$
f_{j}^{-1}(V \cap F(G)) \subset V \cap F(G) .
$$

Proof. Since $G$ satisfies $O S C(U)$, we know that $V_{i} \subset U$. This yields

$$
f_{j}^{-1}\left(V_{i}\right) \subset V_{j}
$$

for each pair $(i, j)$, where $1 \leq i, j \leq m$.

Claim $I \quad J(G) \subset \bar{V}$.

In fact, for any point $w \in V \backslash E(G)$, (2.1) implies that for each $g \in G$,

$$
\left\{g^{-1}(w)\right\} \subset V
$$

Claim $I$ follows from Lemma 2.2.

Claim $I I \quad V \cap F(G) \neq \emptyset$.

Suppose, on the contrary, that $V \cap F(G)=\emptyset$. Then $V \subset J(G)$. Claim $I$ implies

$$
J(G)=\bar{V}=\bigcup_{i=1}^{m} \overline{V_{i}} .
$$

We deduce from Lemma 2.3 that 


$$
\bigcup_{i=1}^{m} \overline{V_{i}}=\bigcup_{j=1}^{m} f_{j}^{-1}\left(\bigcup_{i=1}^{m} \overline{V_{i}}\right)
$$

Lemma 2.5 shows that $\bigcup_{j=1}^{m} f_{j}^{-1}\left(\bigcup_{i=1}^{m} \overline{V_{i}}\right)$ is a proper subset of $\bigcup_{i=1}^{m} \overline{V_{i}}$. From (2.2), this is a contradiction. Our second Claim is proved.

The inclusion $f_{j}^{-1}(V \cap F(G)) \subset V$ for each $j(1 \leq j \leq m)$ is obvious. Now we come to show that

$$
f_{j}^{-1}(V \cap F(G)) \subset F(G) .
$$

Suppose that there exist a point $z \in V \cap F(G)$ and some $k(1 \leq k \leq m)$ such that $f_{k}^{-1}(z) \in J(G)$. Then

$$
f_{k}^{-1}(z) \in f_{k}^{-1}(V) \cap J(G) .
$$

On the other hand, Lemma 2.3 shows that

$$
f_{k}^{-1}(z) \in \bigcup_{i=1}^{m}\left(f_{i}^{-1}(J(G)) \cap f_{k}^{-1}(V)\right) .
$$

Since $G$ satisfies $O S C(U)$, we have

$$
f_{i}^{-1}(V) \cap f_{j}^{-1}(V)=\emptyset
$$

for each pair $(i, j)$, where $i \neq j$ and $1 \leq i, j \leq m$.

Hence

$$
f_{i}^{-1}(\bar{V}) \cap f_{j}^{-1}(V)=\emptyset .
$$

Then Claim $I$ implies that

$$
f_{k}^{-1}(z) \in f_{k}^{-1}(J(G)) \cap f_{k}^{-1}(V),
$$

i.e.

$$
f_{k}^{-1}(z) \in f_{k}^{-1}(J(G) \cap V) .
$$

We see that $z \in J(G) \cap V$. This contradiction completes the proof. 


\section{The proof of Theorem 1.1}

Let $V$ be as in Lemma 2.5, and let $\Omega=V$. The proof follows from Lemmas 2.4 and 2.6.

\section{References}

[1] A. F. Beardon, Iteration of Rational Functions, Springer-Verlag, New York, 1991.

[2] D. A. Boyd, Dynamics and measures for semigroups of rational functions, PhD thesis, University of Illinois, 1998.

[3] D. A. Boyd, An invariant measure for finitely generated rational semigroups, Complex Variables, 39 (1999), 229-254.

[4] D. A. Boyd and R. Stankewitz, Dynamics of rational semigroups, Lecture notes based on mini-course given at Georg-August-Universität Góttigen in June 22-July 2, preprint.

[5] T. Harada, The dynamics of nearly abelian polynomial semigroups, Proc. Japan Acad., 73 (1997), 36-37.

[6] A. Hinkkanen and J. Martin, The dynamics of semigroups of rational functions I, Proc. London Math. Soc., 73 (1996), 358-384.

[7] A. Hinkkanen and J. Martin, Julia sets of rational semigroups, Math. Zeit., 222 (1996), 161-169.

[8] A. Hinkkanen and G. J. Martin, Some properties of Semigroups of rational functions, In XVIth. Rolf Nevanlinna Colloquim (Joensuu, 1995), de Gruyter, Berlin, (1996),53-58.

[9] J. Milnor, Dynamics in one complex variable: Introductory lectures, Stony Brook Institute for Mathemiatical Science, 1990.

[10] R. Stankewitz, Completely invariant Julia sets of polynomial semigroups, Proc. Amer. Math. Soc., 127 (1999), 2889-2898.

[11] R. Stankewitz, Completely invariant sets of normality for rational semigroups, Complex Variables Theo. Appl., 40 (2000), 199-210. 
[12] R. Stankewitz, Uniformly perfect sets, rational semigroups, Kleinian groups and IFS's,Proc. Amer. Math. Soc., 128 (2000), 2569-2575.

[13] R. Stankewitz, Completely invariant Julia sets of rational semigroups, $\mathrm{PhD}$ thesis, University of Illinois, 1998.

[14] R. Stankewitz, T. Sugawa and H. Sumi,Some counterexamples in dynamics of rational semigroups, Ann. Acad. Sci. Fenn. Math., 29 (2004), 357-366.

[15] N. Steinmetz, Rational Iteration: Complex analytic dynamical systems, de Gruyter, Berlin, 1993.

[16] H. Sumi, On Dynamics of Sub-hyperbolic and Semi-hyperbolic rational semigroups and skew products, Ergod. Th. and Dynam. Sys., 21 (2001), 563-603.

[17] H. Sumi, On Dynamics of Hyperbolic Rational Semigroups, J. Math. of Kyoto Univ., 37 (1997), 717-733.

[18] H. Sumi, On Hausdorff dimension of Jilia sets of hyperbolic rational semigroups, Kodai Math. J., 21 (1998), 10-28.

[19] H. Sumi, Dimensions of Julia sets of expanding rational semigroups, Kodai Math. J., 28 (2005), 390-422.

[20] H. Sumi, Skew product maps related to finitely generated rational semigroups, Nonlinearity, 13 (2000), 995-1019.

[21] Y. Sun and C. Yang, On the connectivity of the Julia set of a finitely generated rational semigroups, Proc. Amer. Math. Soc., 130 (2001), 49-52.

[22] W. Yang, Y. Li and Z. Gong, The random dynamical system formed by finitely many rational functions, Advances In Mathematics(China), $\mathbf{3 3}$ (2004), 447-452.

[23] X. Wang and Zh. Huang, On nearly abelian polynomial semigroups, Proc. Amer. Math. Soc., 133 (2005), 83-89. 
